\title{
INFLUENCE OF VITAMIN D BINDING PROTEIN AND C3-EPIMER ON ACCURACY OF 250H VITAMIN D ELISA ASSAYS
}

N. Heureux*, F. Mathieu, M. Hars DIAsource Immunoassays, Louvain-la-Neuve, Belgium

\section{Introduction}

The measurement of $25 \mathrm{OH}$ Vitamin $\mathrm{D}$ has tremendously evolved since the first competitive protein-binding assay that used tritiated $25 \mathrm{OH}$ Vitamin D3 and a Vitamin D binding protein (DBP) originating from rat. As an example solvent extraction, which the role was to solubilize the hydrophobic $25 \mathrm{OH}$ Vitamin $\mathrm{D}$ and to precipitate its binding proteins, gradually gave way to methods involving $\mathrm{pH}$ shifts, enzymatic digestion, displacement chemicals or hybrid solutions. In 2012, DIAsource Immunoassays launched an innovative ELISA assay. The innovation lies in the release of $25 \mathrm{OH}$ Vitamin $\mathrm{D}$ from its binding proteins that takes place directly in the coated microplate, while all other non-automated assays involve one or multiple preparation steps prior to the immunoassay. The benefits are a decrease in the assay hands-on time, the possibility to fully automate the assay on ELISA platforms and the suppression of the risk for errors due to mislabeling of the dilution tubes. Furthermore the assay is based on patented monoclonal antibodies, which provide the assay an excellent specificity profile and lot-to-lot reproducibility.

Although the acceptance of the Thienpont's and NIST LC-MS/MS assays as reference methods, and the recent initiative towards standardization of the Vitamin D assays [1] have contributed to an improvement in the method-related variability in serum $25 \mathrm{OH}$ Vitamin D measurement, differences remain between assays. These variations can affect the $25 \mathrm{OH}$ Vitamin D clinical decision-making $[2,3]$. The variance in concentration of Vitamin D Binding Protein (DBP) and C3-epi-25OH Vitamin D can both participate in the inter-assay heterogeneity.

\section{Vitamin D Binding Protein (DBP)}

One potential source of assay-related variability lies in the extended physiological range of concentration of DBP. The concentration of DBP is not constant and can be influenced by a number of factors including obesity [4], pregnancy [5], the use of oral contraceptives [6], HRT [7], liver disease [8], renal disease [9-11], proteinuria [12] and intensive care [13]. As one of the major challenge in measuring $250 \mathrm{OH}$ Vitamin $D$ is the displacement of $250 \mathrm{H}$ Vitamin D from DBP, such a wide range of physiological DBP concentrations could influence the accuracy of the several $250 \mathrm{OH}$ Vitamin D immunoassays. This was demonstrated by Heijboer et al. in a recent article in which the accuracy of six $250 \mathrm{OH}$ Vitamin D assays was evaluated across the range of DBP concentration [14].

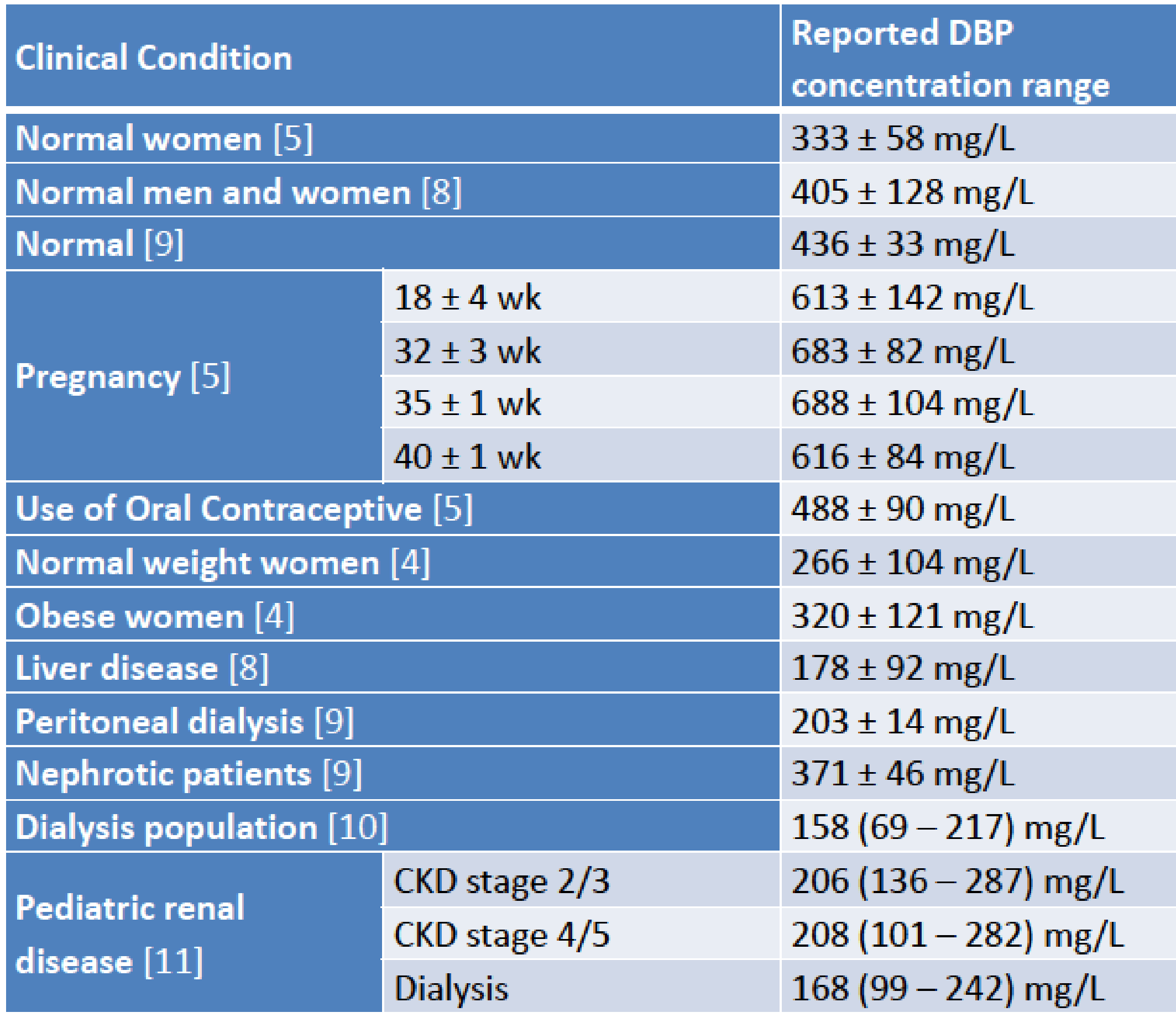

\section{3-epi-25OH Vitamin D (C3-epimer)}

The relevance of 3-epi-250H Vitamin D for Vitamin D physiological actions is still unclear and there is a current general consensus that $3-\mathrm{epi}-25 \mathrm{OH}$ Vitamin D should not be included in the measurement of $250 \mathrm{OH}$ Vitamin D [15]. The concentration of 3-epi-250H Vitamin D, its prevalence and the ratio to $25 \mathrm{OH}$ Vitamin D vary according to populations and reports [1520]. It is generally accepted that 3-epi-25OH Vitamin D is more present in infant and pediatric populations than in adults.

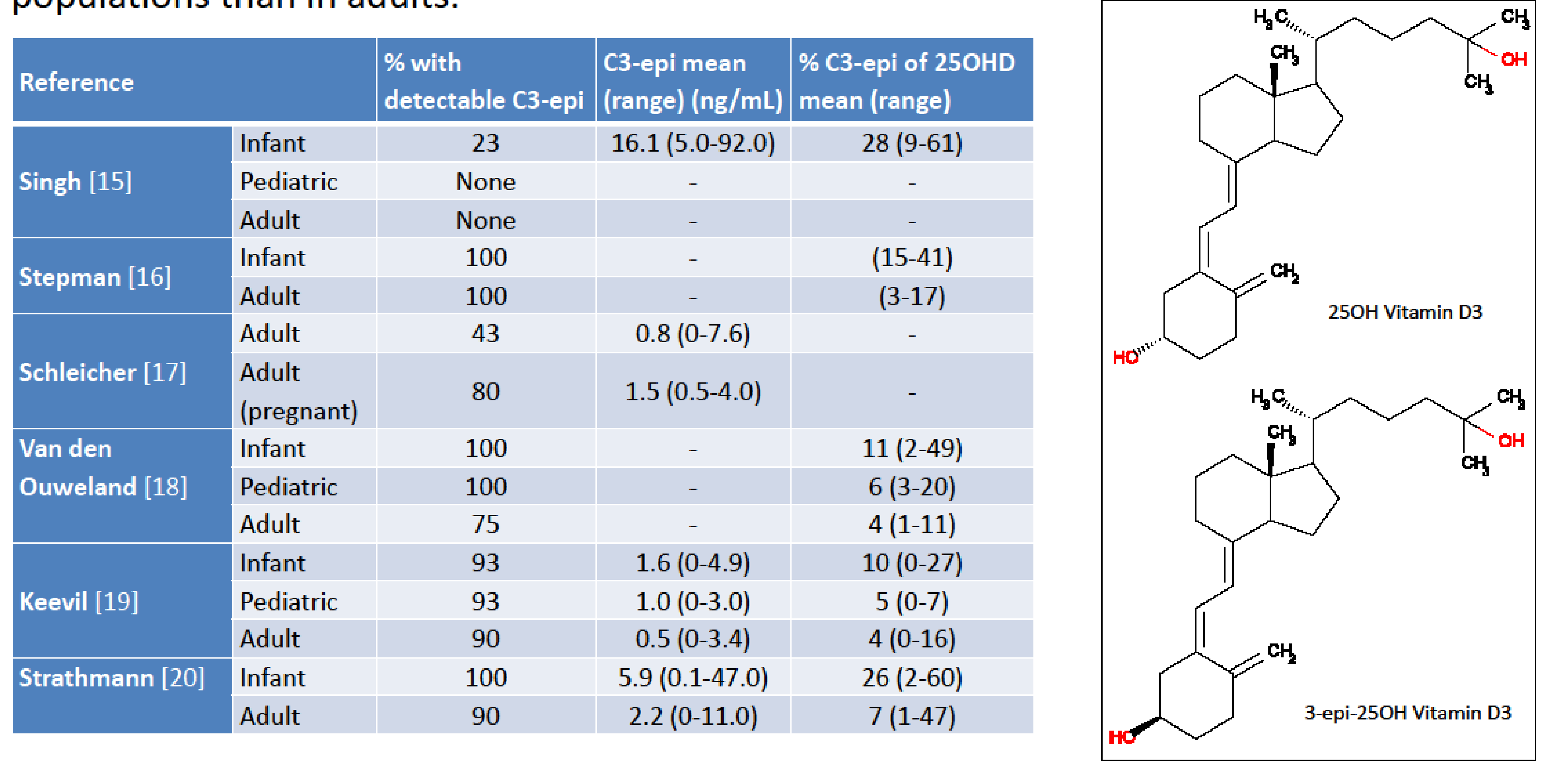

\section{References}

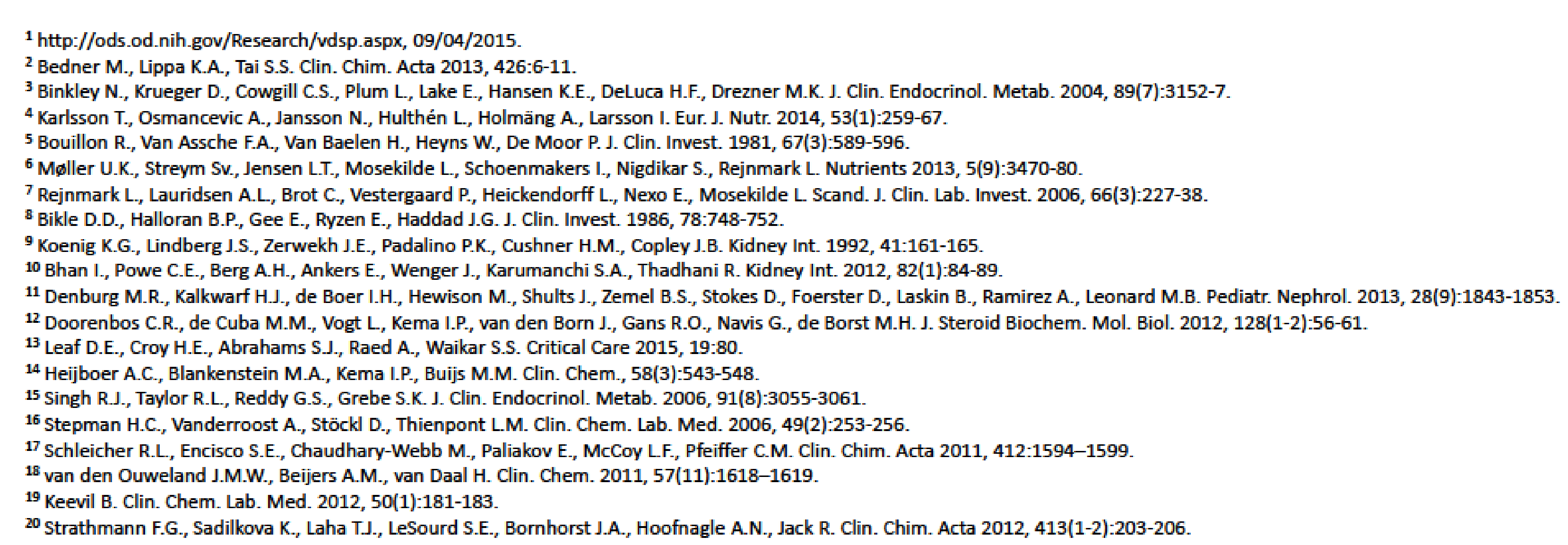

\section{Study Design}

In order to determine the influence of DBP and 3-epi-25OH Vitamin D on several $250 \mathrm{OH}$ Vitamin D ELISA assays, a study examining DBP and 3-epi-25OH Vitamin D as an endogenous interference, similar to how hemoglobin, cholesterol, or total protein would be measured, was performed at the DIAsource facilities.

Five human serum pools containing increasing concentration of $250 \mathrm{H}$ Vitamin D (range 8-60 ng/mL) were prepared from native, single patient, serum samples. Each of the five serum pools were divided into aliquots, and DBP and C3-epimer were spiked into aliquots of each pool to various levels. $25 \mathrm{OH}$ Vitamin D measurements of spiked and unspiked pools were then performed using different ELISA assays. All measurements were performed on the same day, using the same aliquots.

The following $25 \mathrm{OH}$ Vitamin D assays were evaluated

- DIAsource 25OH Vitamin D Total ELISA

- Euroimmun 25-OH Vitamin D ELISA

- Calbiotech 25-OH Vitamin D ELISA

- DRG 25-OH Vitamin D (total) ELISA

- IDS 25-Hydroxy Vitamin D EIA

Immundiagnostik 25-OH Vitamin D direct, ELISA

\section{Results}

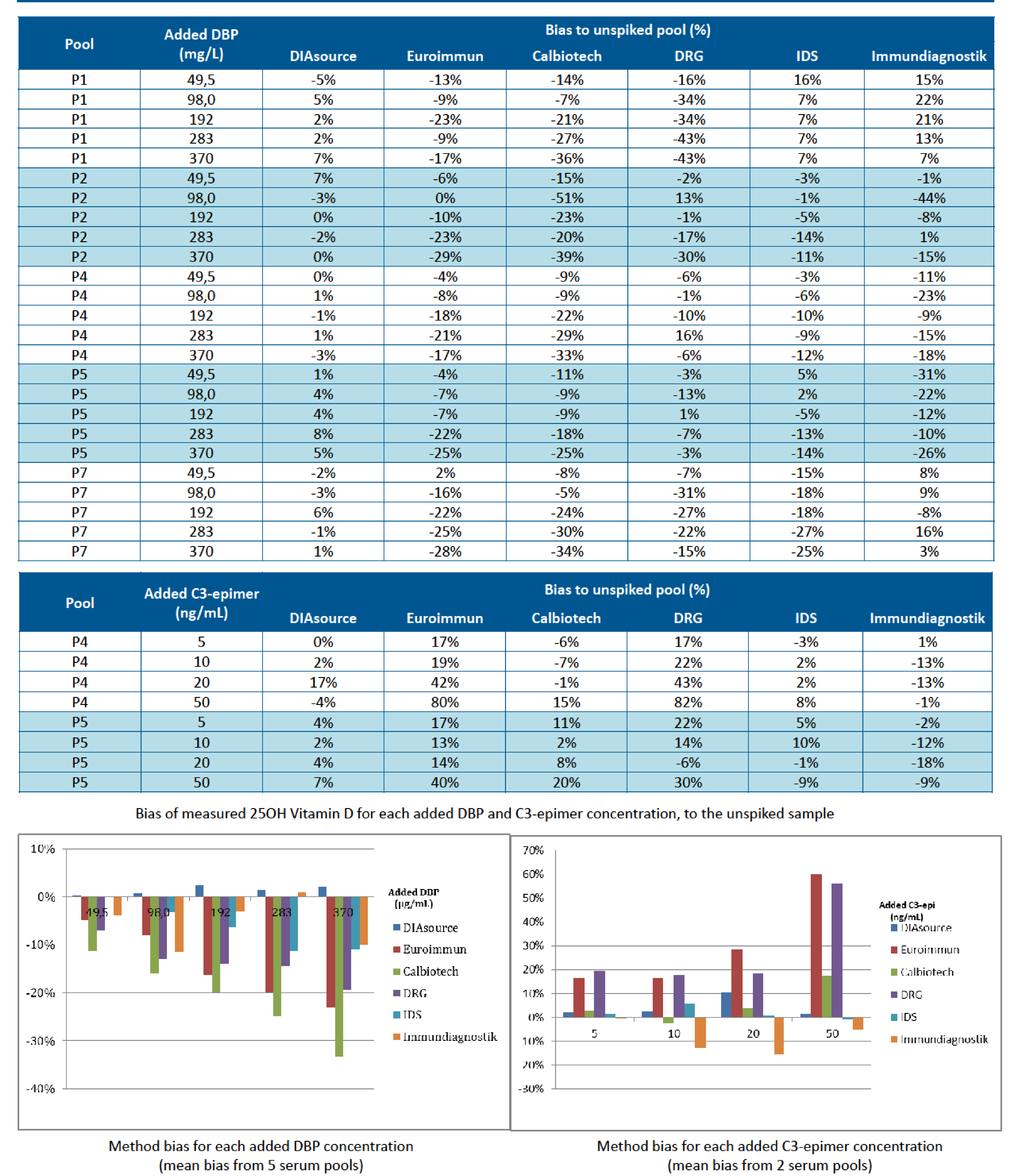

\section{Discussion}

The DIAsource $250 \mathrm{OH}$ Vitamin D Total ELISA assay was the only method that was not influenced nor by the added DBP, neither by the added C3-epimer.

The IDS ELISA assay was slightly negatively influenced by the addition of DBP but not by the addition of C3-epimer. The Calbiotech assay showed a strong influence upon addition of exogenous DBP but was not significantly impacted by the addition of C3-epimer. The DRG and Euroimmun methods were both strongly affected by the addition of both DBP or C3-epimer.

The different $250 \mathrm{H}$ Vitamin $\mathrm{D}$ release methods and the antibody specificity profile, or DBP specificity profile in the case of the DRG assay, are mostly responsible for these contrasting behaviors. 